\title{
Valoración de la salud mental de los estudiantes de enfermería según su percepción. FENOB- UNA. San Lorenzo, 2019
}

\author{
Marian Ynés Cardozo Florentín'
}

\begin{abstract}
Resumen
Introducción: La salud mental es un estado de bienestar en el cual el individuo es consciente de sus propias capacidades, puede afrontar las tensiones normales de la vida, trabajar de forma productiva y ser capaz de contribuir a su comunidad. Se puede afirmar que las funciones mentales están interconectadas con el funcionamiento físico, social y con el estado de salud general. Es decir, la salud mental es el producto de todos los aspectos de la vida cotidiana del individuo. Si la interacción del individuo con su medio es inadecuada, se alteran los patrones de normalidad y, por ende, coloca al hombre en una situación de vulnerabilidad, y que en cualquier momento de su vida puede desembocar en un problema de salud mental. Los estudiantes de enfermería no están exentos de padecer este tipo de problemas, debido a que los mismos están expuestos a niveles de estrés más elevados que la población general.
\end{abstract}

Objetivo:Determinar la valoración de la salud mental de los estudiantes de Enfermería según su percepción. FENOB-UNA. San Lorenzo. 2019

Material y Método: El diseño de estudio fue observacional, descriptivo, de corte transversal con enfoque cuantitativo. La muestra estuvo conformada por 232 estudiantes de la carrera de enfermería. El muestreo fue probabilístico sistemático. El método y la técnica fue la encuesta autosuministrada. Los instrumentos utilizados fueron el Cuestionario de Salud General de GoldbertGHQ28 y el Cuestionario de Prueba de Detección de Consumo de Alcohol, Tabaco y Sustancias-ASSIST; que contenían preguntas cerradas de selección múltiple. Resultados: El grupo etario predominante fue de $17-21$ años $(70,69 \%)$, con predominio del sexo femenino (73,71\%). Los principales diagnósticos hallados fueron que el 58,62\% de los estudiantes consume alcohol, el 22,84\% presenta ansiedad e insomnio, el 18,97\% síntomas somáticos, el 8,19\% depresión grave y el $6,47 \%$ consume drogas ilícitas.

Conclusión: De acuerdo con lo señalado y los resultados demostrados, un

1. Universidad Nacional de Asunción. Facultad de Enfermería y Obstetricia, Paraguay.

E-mail: mariancardozoflorentin22215@gmail.com

DOI: $10.26885 /$ rcei.foro.2019.247

Trabajo publicado en acceso abierto bajo Licencia Creative Commons. 
número representativo de estudiantes de la Carrera de Enfermería de la Facultad de Enfermería y Obstetricia de la Universidad Nacional de Asunción, se encuentra dentro de los parámetros normales de salud mental. Sin embargo, los índices de ansiedad e insomnio, síntomas somáticos y consumo de alcohol son elevados. Aunque el consumo de sustancias ilícitas no lo es, resulta inquietante que este tipo de sustancias se encuentren entre las opciones de uso de los futuros profesionales de la salud.

Un punto a considerar para otros trabajos en esta línea de investigación es realizar un análisis de factores asociados que pudieran influir de manera positiva o negativa en la salud mental de los estudiantes de enfermería.

Realizar este tipo de trabajo de investigación con estudiantes del área de salud, permite identificar las condiciones mentales en que se encuentran los universitarios durante su proceso de formación profesional y futuro lanzamiento al mercado laboral.

Palabras clave: salud mental, estudiantes universitarios, enfermería.

\section{Referencias}

Antúnez, Z., Vinet, E. V. (2013). Problemas de salud mental en estudiantes de una universidad regional chilena. Rev Med Chil, 141(2), 209-16. http://www.scielo.cl/scielo.php?script=sci arttext\&pid=S0034-98872013000200010\&lng=en\&nrm=iso\&tlng= en

Bertolote, J. M. Raíces del concepto de salud mental. World Psychiatry (Ed Esp), 6(2), 113-6. https://es.scribd.com/doc/63126150/ Origen-del-concepto-de-Salud-Mental

Padilla, M. L., Rojo, A. P. R., Contreras, J. H., Muñoz, B. A. M. (2010). Salud mental y bienestar psicológico en los estudiantes universitarios de primer ingreso de la Región Altos Norte de Jalisco. Rev Educ y Desarro, 14, 31-7. http://www.cucs.udg.mx/revistas/edu_desarrollo/anteriores/14/014_ Perez.pdf

Pérez-Padilla, E. A., Cervantes-Ramírez, V. M., Hijuelos-García, N. A., PinedaCortés, J. C., Salgado-Burgos, H. (2017). Prevalencia, causas y tratamiento de la depresión Mayor. Rev Biomed, 28(2), 73-98. http:// www.medigraphic.com/pdfs/revbio/bio-2017/bio172c.pdf

Videbeck, S. L. (2012). Enfermería Psiquiátrica (5 ed.). Barcelona: Lippincott Williams \& Wilkins. 